\title{
I flipped my tutorials: a case study of implementing active learning strategies in engineering
}

\author{
Jonathan Verrett ${ }^{1}$, Anne-Marie Kietzig ${ }^{2}$, Maria Orjuela-Laverde ${ }^{3}$ \\ ${ }^{1,2,3}$ Faculty of Engineering, McGill University, Montreal, QC, Canada \\ 1.jonathan.verrett@mail.mcgill.ca, ${ }^{2}$ anne.kietzig@mcgill.ca, ${ }^{3}$ maria.orjuela-laverde@mcgill.ca
}

\begin{abstract}
A$ variety of active learning strategies have been applied to engineering classrooms, including flipping classrooms by recording lectures and having students watch them outside of class time. In this study, a similar approach was used for long-answer problems presented in one-hour tutorial sessions. Problem solutions were recorded and made available online. Instead of solving long-answer problems, tutorials began with a review of relevant material. The review was then followed by independent working time where students were free to interact with the teaching assistant and discuss concepts with one another while working on an online quiz.
\end{abstract}

Students generally responded very positively to the changes and appreciated the ability to go through problem solutions at their own pace with the recordings. In tutorials, the quizzes were successful at encouraging discussion of course content amongst students. The techniques also provided a repository of online videos and quizzes to be used in future course iterations.

Keywords: Flipped Classroom, Online Quizzes, Active Learning, Tutorials, Teaching Assistant, Chemical Engineering

\section{INTRODUCTION}

The Faculty of Engineering at McGill University founded the Teaching Enhancement Initiative (TEI) in the winter semester of 2013 to enhance teaching and learning within the faculty. To date, more than 50 professors and 60 teaching assistants from the different departments within the Faculty have participated.

Faculty members have been invited to lunch sessions designed to introduce teaching strategies that are appropriate for engineering courses at McGill given factors such as class size, year level, and specific subject matter. These sessions allow participants to discuss specific teaching techniques and clarify questions about implementation.
Workshops for teaching assistants (TAs) focus on the nature of teaching and learning in a university setting and how to respond to challenges they face within their practice as TAs.

In the same line as Engineering's TEI, the Faculty of Science at McGill formed the Tomlinson Project in University Level Science Education (TPULSE) in July 2002. This initiative seeks to implement current ideas and practices on the teaching of science in undergraduate education. T-PULSE has become a resource for faculty and graduate students who wish to improve their teaching. It is in the context of the TEI and T-PULSE that the flipped tutorial experience was born.

The main goal of the TEI project in the Faculty of Engineering is to enhance student-centered and evidence-based teaching within all departments. The initiative invites instructors and TAs to use active learning strategies as a teaching tool in their courses. Active Learning (AL) has been broadly defined as any instructional strategy that "requires students to do meaningful learning activities and think about what they are doing" [1].

For more than two decades, researchers have been studying the use of AL strategies with undergraduate students across science, technology, engineering and math (STEM) disciplines. In the field of engineering education, Prince et al. (2009) conducted a study to examine engineering students' conceptual change when inquirybased activities were introduced in the curriculum [2]. Results of the study suggest that inquiry-based activities were effective in promoting conceptual change. A metaanalysis of 225 studies conducted by Freeman et al. (2014) indicates that student performance on examinations and concept inventories increases $6 \%$ when using AL strategies compared to traditional lecturing [3]. Studies included in this meta-analysis also reported that students in traditional lecturing courses were 1.5 times more likely to fail compared to students following an $\mathrm{AL}$ approach. 
The flipped tutorial introduced in this paper follows the strategy of the flipped classroom (or flipped learning) that some professors have been using in their classrooms [4]. The principles of flipped learning, as defined by Bergman and Sams (2012), are [5]:

(1) Transferring the ownership of the learning to the students,

(2) Personalizing learning for all students,

(3) Giving teachers time to explore deeper learning opportunities and pedagogies with their students,

(4) Making learning (not teaching) the center of the classroom, and

(5) Maximizing the face-to-face time in the classroom

The objective of this paper is to introduce the flipped tutorial strategy implemented in a chemical engineering tutorial at McGill's Faculty of Engineering. The paper will also describe how this strategy, combined with an online self-paced quiz, increased students' engagement in the course. Results from this experience suggest a positive response from undergraduate students when using these types of AL strategies in their learning environment.

\section{COURSE DESCRIPTION}

CHEE 423 Reactions Engineering is a core course generally taken in the final year of a chemical engineering degree at McGill University. It aims to introduce students to reactor design principles and relies on many previously taught subjects such as heat and mass transfer, physical chemistry and fluid mechanics. CHEE 423 is offered once per year in the fall semester, with 85 students in the fall 2014 offering. The course schedule consisted of 3 onehour lectures as well as 1 one-hour tutorial per week. Tutorials were not always held on a weekly basis, as some tutorials were substituted as classes and vice-versa. Tutorial sessions were used as practice and review sessions after a section of the course content had been taught. During the 13 weeks of the course, 10 tutorials were given.

Previous iterations of the course used short (fiveminute) in-class graded quizzes to identify gaps in conceptual understanding. Tutorials were used to supplement this with ungraded long-answer calculation problems. In previous years, a typical tutorial would consist of a short review of material, followed by a presentation of the solution to a long-answer problem or problems. Efforts were made to engage the students using AL strategies such as think-pair-share while solving the long-answer problems. The quizzes and long-answer problems were effective at giving students opportunities for low-stakes assessment but became repetitive year after year. Students soon had the answers to quizzes from previous years and with a limited number of types of short-answer questions, it seemed unfair to grade students on the quizzes if some had solutions and others did not. The tutorial long-answer problems were also not engaging as many students as hoped. These issues provided the impetus to re-structure the tutorial time in the course.

During the Fall 2014 term, the long-answer problems were removed from the tutorial and were recorded and made available as instructional videos with solutions, using online course management software. 10 videos were created using the long-answer problems from previous years. Videos were recorded using DoodleCast Pro, a screen-capture application for ipad that also includes sound-capture. Initially questions were broken into various parts and videos were recorded in segments between 5 and 20 minutes. Students however preferred having a single video file, as this was simpler to download and manage since the online course management software used did not offer streaming video.

The revised tutorial structure consisted of a review of material for the first 15 minutes followed by an open space to ask the TA and other classmates questions and/or work on a quiz based on the review material. Quizzes were hosted on the online course management software and were always linked to a specific tutorial and section of course content. Quizzes consisted of no more than five (5) questions focused on conceptual and short calculation problems. Because the quizzes were in digital format, questions focused on matching concepts to diagrams, numerical solutions, true/false, multiple choice and other types of questions that could be machine graded. The students had unlimited attempts on the quiz and were strongly encouraged to work with other students before seeking out the TA for help. Answers were randomized where possible to ensure quizzes were different between students, so that students were required to understand concepts rather than simply copy from their neighbour. In an attempt to ensure students kept pace with course materials, a grade of $80 \%$ was required on a quiz before being granted access to the following quiz.

\section{STUDENT SURVEY AND USAGE DATA}

Data for quizzes were recorded and included the date of quiz attempts and score. Data were not collected on video viewing due to the limitations of the course management software.

At the last class of the semester, students were asked to complete an anonymous online survey with questions on both the long-answer videos and the online quizzes. The survey included eight (8) Likert scale questions containing the responses "Strongly agree", "Agree", "Neutral", "Disagree" and "Strongly disagree". Questions 
used for both long-answer videos and online quizzes are presented together for brevity.

(i) The use of (long-answer videos/online quizzes) contributed to learning the subject matter.

(ii) (Long-answer videos/online quizzes) helped me prepare for the midterms/final exam.

(iii) (Long-answer videos/online quizzes) encouraged me to discuss course content with my peers.

(iv) Long-answer videos helped me focus on what I should be learning in the course.

(v) I was more actively engaged with the class lectures and tutorials because of the online quizzes.

The survey also included five (5) open-ended questions presented below.

1. What recommendations would you give to other instructors using (long-answer videos/online quizzes)?

2. What other courses would benefit from the strategy of (long-answer videos/online quizzes)?

3. Do you have any other comments, questions or concerns?

Since students typically have other classes where long-answer problems are solved in tutorial, another question and comment section assessed their preference between online and in-tutorial problem solving. Students were given the option to state whether their learning would be "less", "the same" or "better" with long-answer problems solved in tutorials and also asked to comment on their preference.

\section{STUDENT PERSPECTIVE}

\subsection{Long-answer videos}

Sixty-seven (67) of the 85 students in the course responded to the survey. Results were grouped into positive ("Agree" or "Strongly agree"), neutral, or negative ("Disagree" or "Strongly Disagree". The vast majority, $91 \%$, of students found the long-answer videos positively contributed to learning the course material. Furthermore, $88 \%$ found that they helped them study for exams and $75 \%$ indicated the questions helped them find topics to focus on in the course. Comments from students reflect these results and offer advice for improvement mainly to focus on exam-type questions rather than on questions involving solutions using computational software.

These results are promising, but the main thrust of using online videos was that students could review the videos and watch them at their own pace. A large percentage $(84 \%)$ of students said their learning would be less with an in-class long-answer problem solution session (thus preferring the video tutorials) with another $12 \%$ stating both delivery methods have the same impact. Many students noted the advantage of being able to work through the videos at their own pace, which helped their understanding. Students also enjoyed being able to focus on conceptual problems, review and interaction in the tutorial. One worry was that the videos might increase student workload. However, students generally report using the videos to structure their independent study and very few stated issues with additional time constraints. Videos were somewhat successful at stimulating student discussions with their peers, with $40 \%$ indicating the questions contributed to discussion and $46 \%$ neutral. Since some students may have watched the videos while studying alone, they may not have any additional benefit for interaction as compared to assigning practice problems. Overall the videos were well received and appreciated by students, with the most notable aspect being that they could follow them at their own pace.

\subsection{Quizzes}

The online quizzes aimed to give students the opportunity to test their conceptual understanding of course material. Quizzes were generally well received by students with $80 \%$ of students reporting quizzes were successful at contributing to their learning the subject matter and $70 \%$ finding they helped to study for exams. Aggregate marks students received on the quizzes (with all quizzes weighted equally) were compared to their final exam grades in figure 1. No clear trend is present, but students with higher quiz marks generally performed better on exams. Whether these students performed better due to quizzes, or were simply more prepared for exams was not assessed.

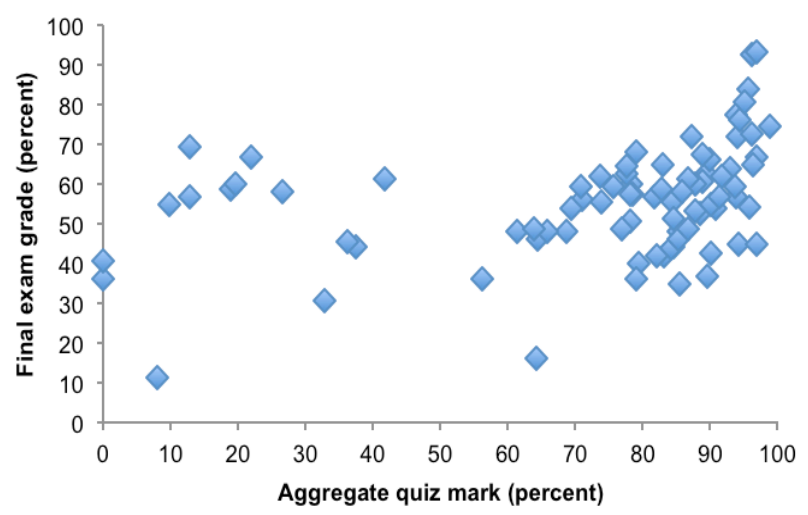

Fig. 1. Student final exam grades and aggregate quiz marks (with quizzes weighted equally) in percent.

The numbers of students completing the quizzes (achieving a score greater than $80 \%$ ) before the next tutorial varied, but generally declined over the course of 
the term, as can be seen in figure 2. The numbers of students completing the quizzes before the final exam were much higher, but also decreased with increasing quiz number. Notable poor performers were the last two quizzes. Most quizzes required completion of previous quizzes before allowing access; however quiz 8 had no such restriction, possibly reducing the impetus to solve quiz 7 . Since quizzes were only graded once submitted in full, and not on a question-by-question basis, students may have begun focusing their study on the questions most relevant to them and not worrying about the overall quiz grade. Figure 3 shows that quizzes 7 and 8 did not have a significantly smaller number of attempts than other quizzes and were thus likely still used by students for study. Many students commented that a quiz with a question-by-question format with immediate feedback would be more useful for their learning as they could focus on the questions they found difficult rather than needing to re-submit the entire quiz each time. Unfortunately this was impossible to implement with the current course software.

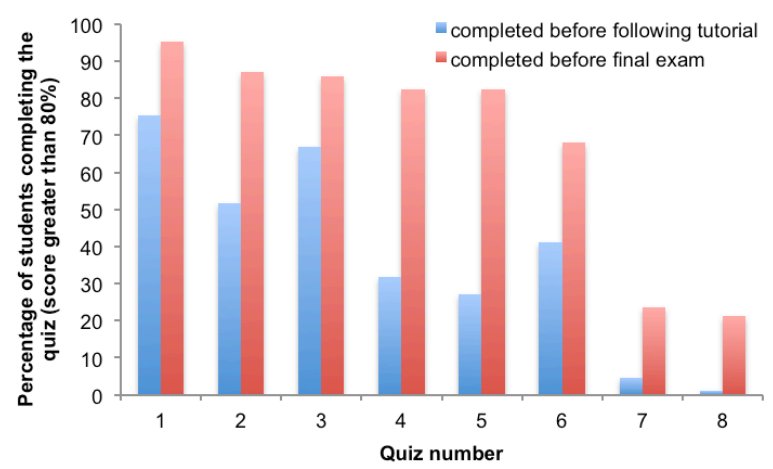

Fig. 2. Number of students having completed a quiz (received a score greater than $80 \%$ ) before the following tutorial (blue) and before the final (red).

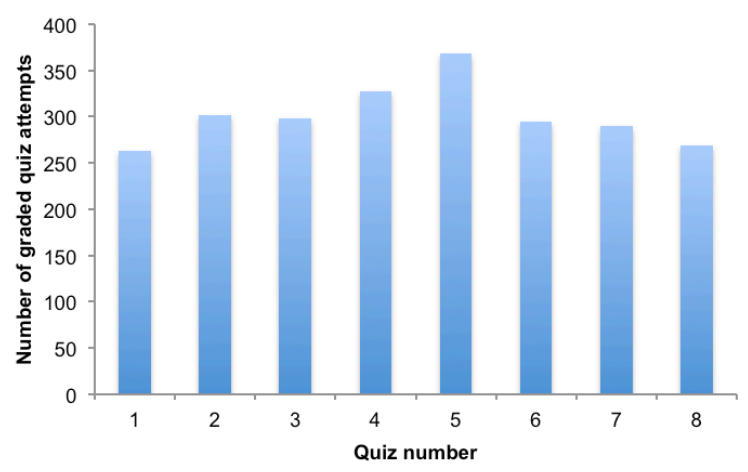

Fig. 3. Number of graded quiz attempts for each quiz.

Quizzes aimed to be used to structure peer learning in the tutorial sessions. $78 \%$ of students found the quizzes encouraged them to discuss course content with their peers, which was much higher than the online videos
(40\%). There was much more discussion during the tutorial time than in previous years when the long-answer problems were being solved. One possible explanation is the self-paced nature of the online quizzes. Students can study at their group's pace and take breaks to answer each other's questions rather than needing to follow at the class' pace. This method may also encourage timid students to interact as they can ask classmates or the TA for help without speaking to the entire class. Despite the feeling that the quizzes encouraged interaction, only $51 \%$ of students found they were more engaged with tutorials due to the quizzes. This may have been due to technical challenges implementing the quiz, causing some frustration for students. Further refining of quiz questions to ensure they are suitable to the online format should help students better engage with the material.

\section{TEACHING ASSISTANT PERSPECTIVE ON IMPLEMENTATION}

Having previously taken the course as an undergraduate in the fall of 2010, I had a good grasp of the content. I had also been the teaching assistant for the past two years, during which time I taught tutorials, held office hours and corrected quizzes. I have also been actively engaged in pedagogy at McGill as a Graduate Teaching Fellow in TPULSE. The fellows take a university course titled "Teaching and Learning in Higher Education" (course code EDPH 689), offered by the Education Department, which gives a background in pedagogy to students outside education. In addition to this, the fellows provide workshops to undergraduate and graduate students at McGill on various topics in teaching and learning. These experiences provided me with a great deal of knowledge about both the course and pedagogy, and led me to propose moving the long-answer videos online. The idea for online quizzes came from discussions with the course instructor (Prof. Kietzig).

Though my background is specialized, my general feeling is that a motivated TA who is comfortable interacting with students and interested in technology would be able to perform similar tasks. The long-answer problems had already been formulated and solved from previous years, meaning much of the setup time was learning to record and edit videos. On average, it took about five hours for each video from start to finish, including understanding and reviewing the question and then recording and editing the video. The quizzes were created in a similar process to the long-answer videos, since they were also based on in-class quizzes from previous years. Most of the time was spent learning to use the online learning platform and adapting questions to this format. Roughly five hours was spent setting up and perfecting each quiz to ensure that they ran smoothly. 
One key factor was working closely with the instructor. She provided the content or focus of the questions, which I could then adapt to the online format. We would check-in on a weekly, if not, more frequent basis to ensure that quiz questions were aligned with course content and responding to concepts that students were finding difficult.

One advantages of using these techniques is that there is more informal contact with students during tutorial time and less of a need to rush through content, since it is now online. Both the instructor and myself found that fewer students came to office hours, possibly because students had more time for contact at the tutorials. These techniques also offer a repository of course-specific online content to build upon in future years. TAs have access to the review content, quizzes and videos for next year, thus helping with TA turnover. In future years, supplemental TA time can be used to create new videos or quizzes, focusing on relevant content suggested by students or the instructor.

\section{CONCLUSIONS}

The strategy to flip tutorials by placing long-answer problem solutions online was generally well received by students. Recommendations for future implementation include the ability to stream and monitor student use of videos. Quizzes were also well received and were successful at structuring interaction time in the tutorials. Questions will need to continue to be improved and adapted to the online format in future iterations. Improvements to the management software to allow students to have feedback on a question-by-question basis would also help. Future TAs will require some training, support and enthusiasm to continue with this strategy, but will probably find it engaging and rewarding should they choose to commit to it.

\section{Acknowledgements}

The authors would like to thank the McGill Teaching Enhancement Initiative for providing funds and support to make the project possible.

\section{References}

[1] M. Prince, "Does Active Learning Work? A Review of the Research," Journal of Engineering Education, vol. 93, pp. 223-231, 2004.

[2] M. J. Prince, M. A. S. Vigeant, and K. Nottis, "A preliminary study on the effectiveness of inquiry-based activities for addressing misconceptions of undergraduate engineering students," Education for Chemical Engineers, vol. 4, pp. 29-41, 2009.
[3] S. Freeman, S. L. Eddy, M. McDonough, M. K. Smith, N. Okoroafor, H. Jordt, and M. P. Wenderoth, "Active learning increases student performance in science, engineering, and mathematics," Proceedings of the National Academy of Sciences, vol. 111, pp. 8410-8415, 2014.

[4] J. L. Bishop and M. A. Verleger, "The flipped classroom: A survey of the research," in ASEE National Conference Proceedings, (Atlanta, GA; June 23-26), 18 pp., 2013.

[5] J. Bergmann and A. Sams, Flip your classroom: Reach every student in every class every day. Arlington, VA: International Society for Technology in Education, 2012, 120 pp. \{ISBN 978-1564-84315-9\} 